\title{
Spin- and Energy-Dependent Tunneling through a Single Molecule with Intramolecular Spatial Resolution
}

\author{
Jens Brede, ${ }^{1}$ Nicolae Atodiresei, ${ }^{2,3, *}$ Stefan Kuck, ${ }^{1}$ Predrag Lazić, ${ }^{2,4}$ Vasile Caciuc, ${ }^{2}$ Yoshitada Morikawa, ${ }^{3}$ \\ Germar Hoffmann, ${ }^{1, \dagger}$ Stefan Blügel, ${ }^{2}$ and Roland Wiesendanger ${ }^{1}$ \\ ${ }^{1}$ Institute of Applied Physics, University of Hamburg, 20355 Hamburg, Germany \\ ${ }^{2}$ Institut für Festkörperforschung and Institute for Advanced Simulation, Forschungszentrum Jülich, 52425 Jülich, Germany \\ ${ }^{3}$ The Institute of Scientific and Industrial Research, Osaka University, Osaka, Japan \\ ${ }^{4}$ Rudjer Boskovic Institute, Zagreb, Croatia \\ (Received 18 March 2010; published 21 July 2010)
}

\begin{abstract}
We investigate the spin- and energy-dependent tunneling through a single organic molecule $(\mathrm{CoPc})$ adsorbed on a ferromagnetic Fe thin film, spatially resolved by low-temperature spin-polarized scanning tunneling microscopy. Interestingly, the metal ion as well as the organic ligand show a significant spin dependence of tunneling current flow. State-of-the-art ab initio calculations including also van der Waals interactions reveal a strong hybridization of molecular orbitals and substrate $3 d$ states. The molecule is anionic due to a transfer of one electron, resulting in a nonmagnetic $(S=0)$ state. Nevertheless, tunneling through the molecule exhibits a pronounced spin dependence due to spin-split molecule-surface hybrid states.
\end{abstract}

DOI: 10.1103/PhysRevLett.105.047204

Molecular based systems are fascinating, yet promising candidates for nanoscale spintronic devices and open viable routes toward quantum computing [1]. Previous experiments on the spin transport through break junctions [2] and spin valves [3] unveil exciting new frontiers of molecular magnetism. Much effort is dedicated to understand the properties of organic-magnetic interfaces. To this end, spatially averaging techniques show substantial spin injection into, as well as long spin-coherent transport throughout films of metal-phthalocyanines [4,5]. However, detailed and quantitative access to different constituents of a single molecule is desirable, though challenging. Scanning tunneling microscopy (STM) is well established as a probe of a local spin [6-13] in an atomically welldefined environment.

Iacovita et al. recently performed a spin-polarized STM (SP-STM) study of a Co-Phthalocyanine ( $\mathrm{CoPc}$ ) in contact with a ferromagnetic cobalt nanoisland [14]. Stacking contrast, spin-dependent scattering, edge states, mesoscopic relaxations as well as the adsorbate induced modification create a complex environment [15] toward understanding the influence of the substrate on molecular magnetism. After careful selection of electronically equivalent Co nanoislands a ferromagnetic exchange interaction between the molecular spin and the cobalt lead was successfully deduced, both theoretically and experimentally.

In this Letter we demonstrate a significant spin polarization for a $\mathrm{CoPc}$ molecule in contact with a ferromagnetic Fe thin film due to molecule-substrate hybridization even though the molecule loses its net spin. As confirmed by SP-STM, an energy- and site-dependent spin polarization from inversion to amplification is resolved on the submolecular scale. State-of-the-art density functional the-
PACS numbers: 85.75.-d, 68.37.Ef, 73.22.-f, 75.50.Xx

ory (DFT), which includes the decisive role of van der Waals (vdW) interactions, reveals both the magnetic and electronic nature of the molecule coupled to the ferromagnetic substrate. Even though the net spin of the molecule is lost due to a transfer of one electron, spin splitting is recovered through the local bonding of molecular orbitals with $\mathrm{Fe} 3 d$ bands.

Simulations were carried out in the DFT [16] formalism with a plane wave implementation as provided by the VASP code [17]. Pseudopotentials used were generated with the projector augmented wave method [18] by using the PBE generalized-gradient exchange-correlation energy functional [19] (GGA). A slab consisting of two Fe and three $\mathrm{W}$ atomic layers, with a $(5 \times 7)$ in-plane surface unit cell $(22.20 \AA \times 22.42 \AA)$ modeled the molecule-surface system. The kinetic energy cutoff of the plane waves was set to $500 \mathrm{eV}$ while the Brillouin zone was sampled by the $\Gamma$ point. In our calculations we employed a Gaussian smearing with a broadening of $0.11 \mathrm{eV}$. Optimized moleculesurface geometries were obtained by relaxing all molecular degrees of freedom and those of the Fe overlayers by including long-range vdW interactions in a semiempirical way $[20,21]$. The threshold of the calculated forces was fixed to $0.001 \mathrm{eV} / \AA$.

Experiments were performed in an ultrahigh vacuum system with an STM operated at $6 \mathrm{~K}$ [22]. In situ chromium coated tungsten tips with out-of-plane spin sensitivity [23] were utilized for all measurements. 1.8 ML Fe was in situ deposited on a W(110) single crystal kept at $400 \mathrm{~K}$. Afterward, the magnetic sample was cooled down to measurement temperature and spin sensitivity was confirmed by imaging the alternating spin-up $(\odot)$ and spin-down $(\otimes)$ domains of the 2 nd layer portion of the Fe film [23]. CoPc 
molecules were in situ thermally sublimated from a homebuilt crucible onto the precooled substrate to ensure spatially well separated molecules.

All STM images were recorded in constant-current mode at the set current $I$ and sample bias voltage $U$. The local spin polarization of the atomic-scale tunnel junction can directly be determined from height variations $\Delta s$ resulting from different magnetization directions of electronically identical areas as introduced by Wiesendanger et al. [24]:

$$
P_{t} P_{s} \cos \theta=\frac{e^{a \sqrt{\phi} \Delta s}-1}{e^{a \sqrt{\phi} \Delta s}+1}=\tanh \frac{a}{2} \sqrt{\phi} \Delta s \approx \overbrace{\frac{a}{2} \sqrt{\phi} \Delta s}^{\Delta s<1 \AA} .
$$

The polarization of tip $\left(P_{t}\right)$ and sample $\left(P_{s}\right)$ are defined as the normalized difference of the energy integrated spin-up and spin-down states $\left(P_{t(s)}:=\rho_{t(s)}^{\odot}-\rho_{t(s)}^{\otimes} ; \rho_{t(s)}^{\odot}+\rho_{t(s)}^{\otimes}=\right.$ 1). $\theta$ is the angle between the respective magnetization directions, $\phi$ the mean local tunneling barrier height, and $a \cong 1 \mathrm{eV}^{-(1 / 2)} \AA^{-1}$.

Figure 1(a) shows a representative 2nd ML area of Fe after deposition of $\mathrm{CoPc}$ molecules. The two dislocation lines indicate the crystallographic axes. Isolated CoPcs adsorb in three well-defined orientations (denoted as $A$, $B$, and $C$ ). The line profile [Fig. 1(b)] along the indicated positions in (a) quantitatively illustrates the height variation within the atomically flat Fe film $(\Delta s=0.3 \AA)$ and the domain-dependent molecular appearance across the orientation $A \mathrm{CoPc}$ on an up and down domain. This variation is due to the relative alignment of tip and sample magnetization being either parallel or antiparallel. While the center of the molecule (Co site) has the same apparent height on both domains $(\Delta s=0 \AA)$, we see a profound contrast at the ligand site $(\Delta s=-0.3 \AA)$. For an up domain the ligand exhibits an increased apparent height relative to the surrounding Fe film, which is in contrast to the apparent height for a molecule on the down domain.

Thereby, orientation $A$ [25] represents a highly symmetric configuration $\left(C_{2 v}\right.$ symmetry) with one molecular axis oriented exactly along the $\langle 001\rangle$ direction. While there are differences in intramolecular contrast amongst the three molecular orientations [Figs. 1(d) to 1(i)], all three configurations show similar domain-dependent contrast variations. In the following, we focus on molecules in configuration $A$ which are not in the vicinity of dislocation lines or domain walls. As the molecular appearance controllably switches under an applied external magnetic field [26], the magnetic contrast can unambiguously be attributed to the spin-polarized local density of states (SPLDOS) of the molecule-surface system.

First-principles calculations within DFT (GGA) clarify the origin of the observed spin dependence. Calculations for the $A$ geometry show the preferred top adsorption, i.e., the $\mathrm{Co}$ atom on top of a surface $\mathrm{Fe}$ atom. Previous results for a Co island revealed an adsorption at the bridge site

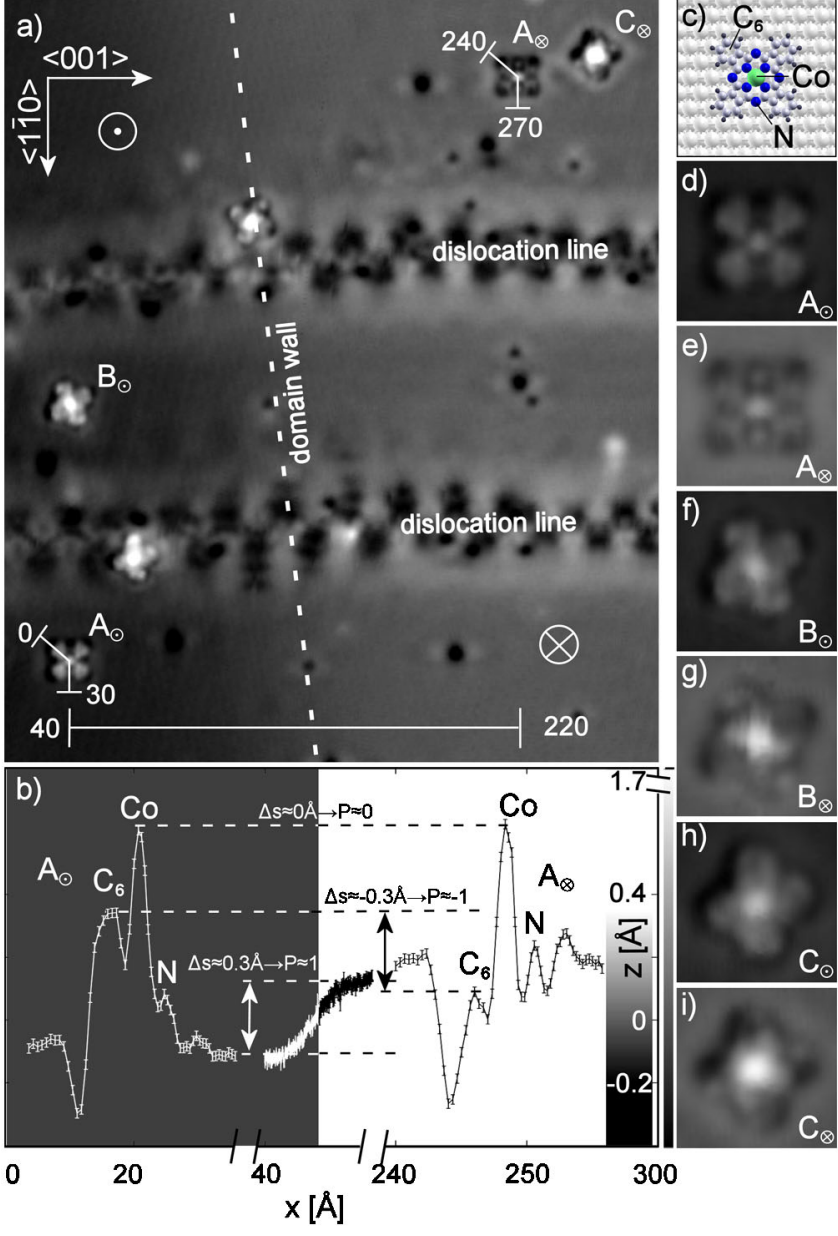

FIG. 1 (color online). (a) SP-STM image of CoPcs on magnetic iron domains $(\odot, \otimes)$. (b) Line profile as indicated in (a). (d)-(i) High-resolution images of CoPcs in all three orientations $(A, B, C)$ on oppositely magnetized domains. (c) Adsorption configuration of a CoPc in orientation $A$. (a) and (d)-(i) $0.05 \mathrm{~V}$, $200 \mathrm{pA}$; (a) $240 \AA \times 280 \AA$; (d)-(i) $25 \AA \times 25 \AA$. The color scale for (a) is nonlinear to illustrate the domain structure, while the color scale for (d)-(i) is linear.

[14], while the hollow site was deduced for a $\mathrm{Au}(111)$ substrate [12]. Furthermore, calculations cover both spinorbit coupling (SOC) as well as the considerable role of $\mathrm{vdW}$ interactions. Results without SOC are discussed in terms of molecular orbitals (MOs) to clearly illustrate the effect of vdW interactions alone.

Figure 2 depicts the change in electronic structure of a $\mathrm{CoPc}$ with increasing molecule-substrate interaction. Figure 2(a) shows the SP-LDOS for the free molecule. In agreement with previous work $[9,12,27,28]$ the origin of spin splitting is an unpaired electron in a MO with $d_{z^{2}}$ contribution situated at the Co site and the total molecular spin $S$ is $\frac{1}{2}$. Figure 2(b) presents the effect of the surface, when vdW forces are neglected during the relaxation process. The molecule adsorbs $3.1 \AA$ above the surface remaining flat. The SP-LDOS shows a hybridization of MOs and substrate $3 d$ states. According to the spatial extension 


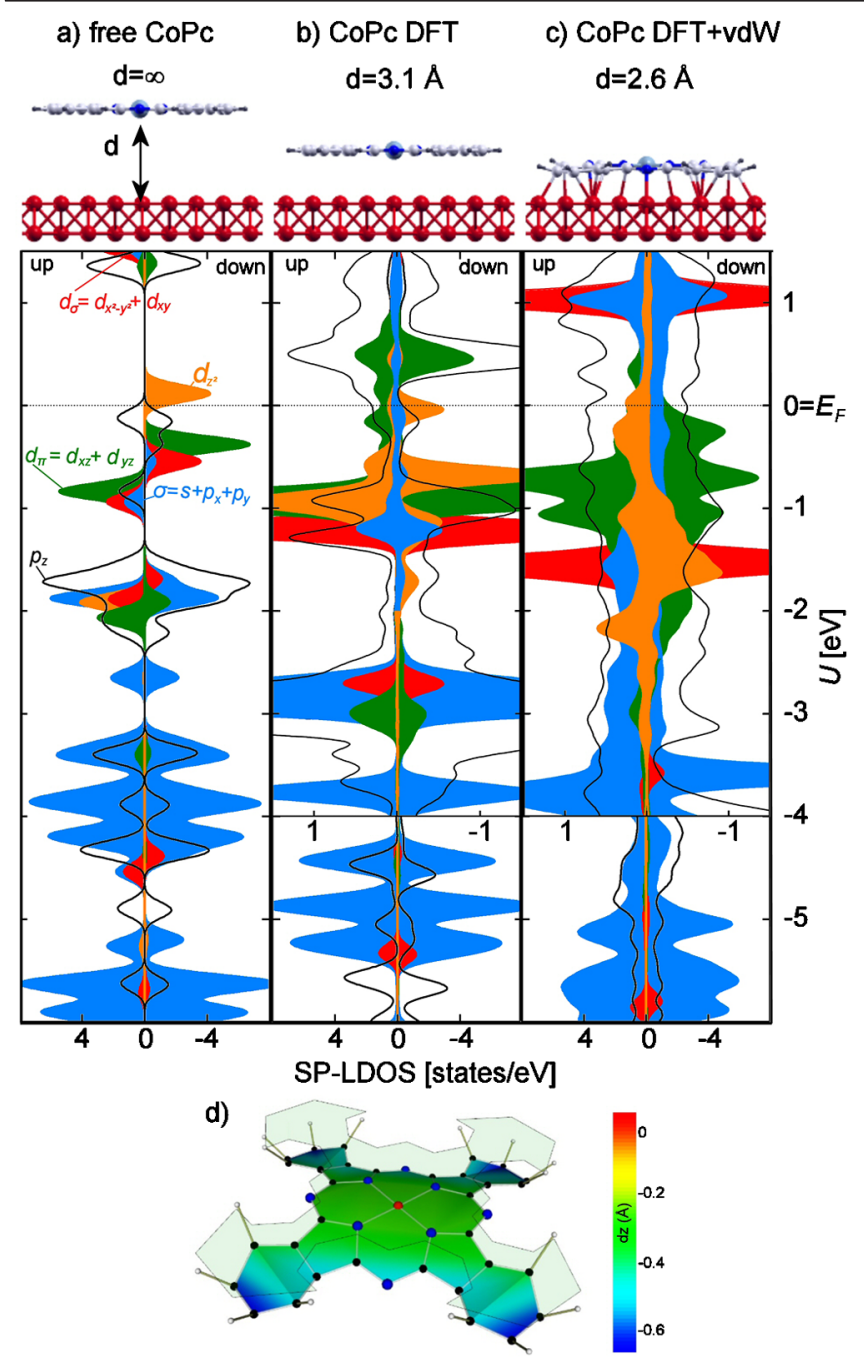

FIG. 2 (color online). The geometry and electronic structure for a free $\mathrm{CoPc}$ (a) and adsorbed on an Fe surface (b) without and (c) with $\mathrm{vdW}$ forces included during the relaxation. (d) Molecular deformation due to vdW forces. The $\mathrm{C}_{6}$ rings and two of the outer $N$ are twisted by up to $0.3 \AA$ toward the surface and away from the plane defined by the Co ion and four inner $N$. All $H$ are pointing away from the surface.

of the MOs perpendicular to the molecular plane (i.e., with $\pi$ character), the hybridization of MOs containing the Co $d_{z^{2}}$ atomic orbital is the strongest, followed by those including $d_{\pi}$ and $p_{z}$ atomic contributions. The $d_{\sigma}$ and $\sigma$ MOs are only slightly broadened, compared to the free molecule case, as they are localized in the molecular plane. The spin splitting of the molecule-surface hybrid states is reduced due to a transfer of an electron from the substrate to the CoPc. As a result, the formerly unoccupied $d_{z^{2}}$ type MO becomes occupied and the total molecular spin is quenched $\left(0 \mu_{b}\right)$.

The role of vdW forces is crucial as it brings the molecule $0.5 \AA$ closer to the surface [Fig. 2(b)] and distorts the molecular geometry [Fig. 2(d)]. This new adsorption geometry has a drastically different electronic structure due to the overall changes of hybridization between molecule and substrate. The SP-LDOS [Fig. 2(c)] shows not only MOs with a $\pi$ character strongly hybridize with spinpolarized Fe $3 d$ states of the same symmetry to form broad spin-split bands but also $\sigma$ type MOs are significantly affected by the interaction with the surface. Again, a transfer of one electron from the surface to the molecule annihilates the molecular spin, but spin splitting is recovered due to the local molecule-surface bonding at different parts of the molecule. The newly formed molecule-surface hybrid states have, within a given energy interval $\left[E, E^{\prime}\right]$, an unbalanced, locally varying electronic charge in the up and down channels which is mapped in SP-STM $\left(E=E_{F}\right.$, $\left.E^{\prime}=E_{F}+e U\right)$.

For a direct comparison of the first-principles calculations with constant-current SP-STM images, isocharge surfaces above the CoPc are extracted from the spatial variation of the energy integrated SP-LDOS. This approach mimics the experimental situation of a local and spin-sensitive tip probing the charge density above the surface at constant current and thereby, accounts for the variation of decay lengths of the different states into vacuum. Figure 3 gathers calculated spin-dependent isocharge surfaces and experimental SP-STM images for $U=$ $0.05 \mathrm{~V}$. The isocharge surfaces reproduce all features of the experimental SP-STM images, only if vdW forces are taken into account [Fig. 3(c)]. Corrections due to the inclusion of spin-orbit coupling effects are minor [Fig. 3(d)].

Figure 4 illustrates quantitatively the local spin polarization. To compensate for the tip polarization, we normalized the experimental polarization above the molecule $\left(P_{\mathrm{CoPc}}^{\exp }\right)$ relative to the spin polarization of the free surface $\left(P_{\mathrm{Fe}}^{\exp }\right)$,

$$
P=\frac{P_{\mathrm{CoPc}}^{\mathrm{exp}}}{P_{\mathrm{Fe}}^{\mathrm{exp}}}=\frac{P_{t} P_{\mathrm{CoPc}} \cos \theta}{P_{t} P_{\mathrm{Fe}} \cos \theta}=\frac{P_{\mathrm{CoPc}}^{\mathrm{sim}}}{P_{\mathrm{Fe}}^{\mathrm{sim}}} .
$$

The normalized polarization $(P)$ is therefore, to a first approximation, independent of the tip polarization and can directly be compared to our simulated polarization $\left(P^{\mathrm{sim}}\right)$.

For comparison, both experimental and theoretical spatial maps of the polarization at three characteristic energies are given in Fig. 4. We see an excellent agreement of

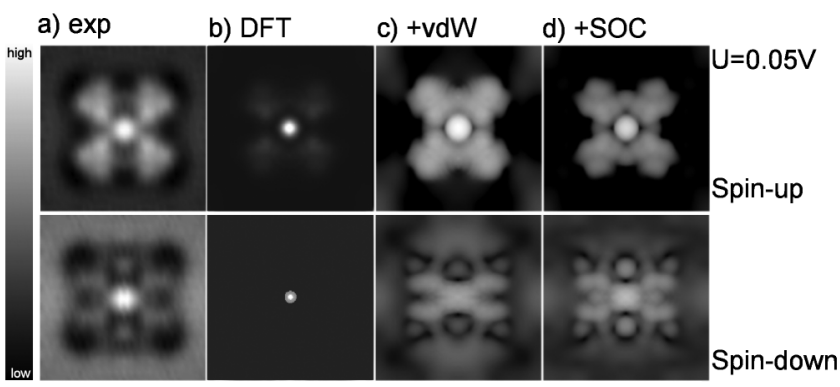

FIG. 3. Experimental and simulated SP-STM images at $U=$ $0.05 \mathrm{~V}$ and $22 \AA \times 22 \AA$ for both spin directions. (a) Averaged experimental images. (b)-(d) Isocharge surfaces; (b) conventional DFT (GGA) (without vdW), (c) DFT + vdW, (d) DFT + vdW and including SOC. 


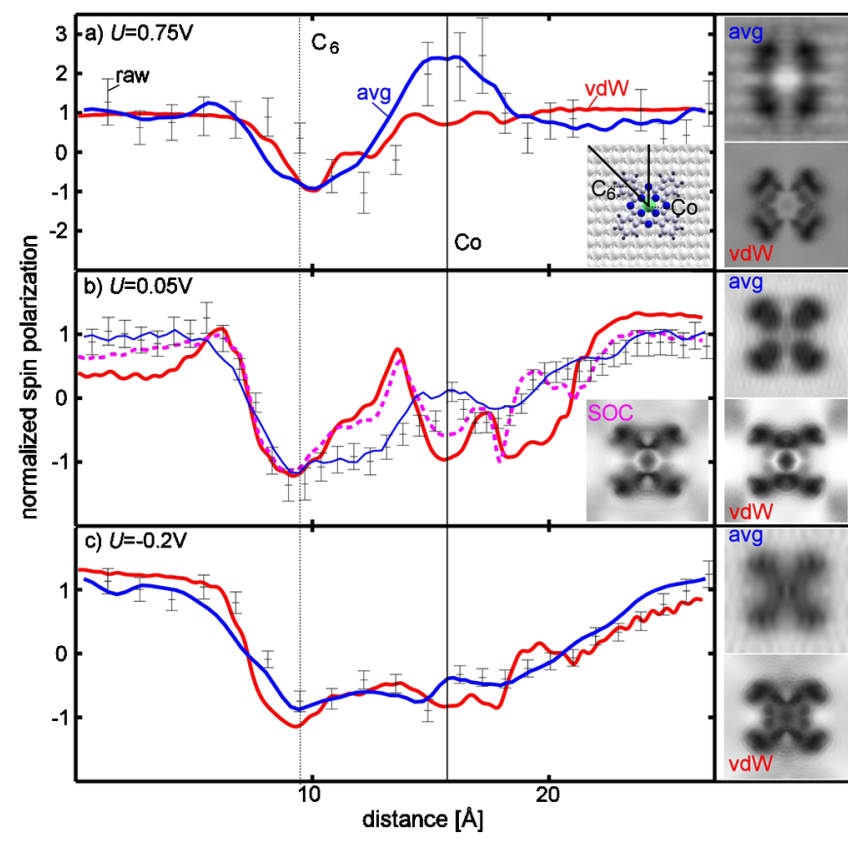

FIG. 4 (color online). Local spin polarization at (a)-(c) three representative energies. Raw and average (over multiple images and according to symmetry [26]) experimental data are compared with DFT simulations incl. vdW and incl. spin-orbit Coupling. Line profiles follow high-symmetry directions as indicated in the sphere model inset in (a). Simulated data including SOC are only given in (b) as the SOC corrections for (a) and (c) are negligible. Insets: $22 \AA \times 22 \AA$.

experimental findings and DFT calculations, when the $\mathrm{vdW}$ relaxed adsorption geometry is used. Remarkably, not only reduction $(1>P>0)$ but also inversion $(P<0)$ and amplification $(P>1)$ of spin polarization can equally be observed. The $P$ above the $\mathrm{C}_{6}$ ring shows an inversion for all energies, while the spin polarization near the Co site ranges from inversion (at $-0.2 \mathrm{~V}$ ) to amplification (at $0.75 \mathrm{~V})$.

In conclusion we investigated single CoPc molecules adsorbed on a ferromagnetic Fe surface and locally observed the energy-dependent spin transport with intramolecular spatial resolution. The tunneling current above the entire molecule - metal ion, as well as the organic ligandshows a high, locally varying spin polarization ranging from inversion up to amplification with respect to the ferromagnetic Fe film. Optimized molecule-surface geometries for the first-principles calculations were obtained by including long-range van der Waals interactions during the relaxation, leading to an excellent agreement of theoretical and experimental data. Therefore, the observed spin polarization is identified as a unique property of the molecule-substrate hybrid states, by our combined DFT and SP-STM approach. Our observations imply that further progress in the field of molecular spintronics does not exclusively rely on tailoring magnetic molecular properties; the clever design of spin-active hybrid interfaces is equally important.
This work is funded by the DFG (SFB 668-A5, SPP1243 and GrK 611), the JSPS, Alexander von Humboldt foundation, the EU project SpiDME. The computations were performed on JUROPA and JUGENE supercomputers at the Jülich Supercomputing Centre, Forschungszentrum Jülich (Germany).

*n.atodiresei@fz-juelich.de

†hoffman@physnet.uni-hamburg.de

[1] L. Bogani and W. Wernsdorfer, Nature Mater. 7, 179 (2008); M. N. Leuenberger and D. Loss, Nature (London) 410, 789 (2001).

[2] J. Park et al., Nature (London) 417, 722 (2002).

[3] A. J. Drew et al., Nature Mater. 8, 109 (2009).

[4] T. Suzuki, M. Kurahashi, X. Ju, and Y. Yamauchi, J. Phys. Chem. B 106, 11553 (2002); T. Suzuki, M. Kurahashi, and Y. Yamauchi, ibid. 106, 7643 (2002).

[5] M. Cinchetti et al., Nature Mater. 8, 115 (2009).

[6] F. Meier, L. Zhou, J. Wiebe, and R. Wiesendanger, Science 320, 82 (2008).

[7] C. F. Hirjibehedin et al., Science 317, 1199 (2007).

[8] Y. Yayon et al., Phys. Rev. Lett. 99, 067202 (2007).

[9] X. Chen et al., Phys. Rev. Lett. 101, 197208 (2008); Y.-S. Fu et al., ibid. 103, 257202 (2009).

[10] N. Tsukahara et al., Phys. Rev. Lett. 102, 167203 (2009).

[11] D. Wegner et al., Phys. Rev. Lett. 103, 087205 (2009).

[12] A. Zhao et al., Science 309, 1542 (2005).

[13] T. Balashov et al., Phys. Rev. Lett. 102, 257203 (2009).

[14] C. Iacovita et al., Phys. Rev. Lett. 101, 116602 (2008).

[15] O. Pietzsch, A. Kubetzka, M. Bode, and R. Wiesendanger, Phys. Rev. Lett. 92, 057202 (2004); O. Pietzsch et al., ibid. 96, 237203 (2006); M. V. Rastei et al., ibid. 99, 246102 (2007); M. Sicot et al., Phys. Rev. B 77, 035417 (2008); H. Oka et al., Science 327, 843 (2010).

[16] P. Hohenberg and W. Kohn, Phys. Rev. 136, B864 (1964).

[17] G. Kresse and J. Hafner, Phys. Rev. B 49, 14251 (1994); G. Kresse and J. Furthmüller, ibid. 54, 11169 (1996).

[18] P.E. Blöchl, Phys. Rev. B 50, 17953 (1994).

[19] J. P. Perdew, K. Burke, and M. Ernzerhof, Phys. Rev. Lett. 77, 3865 (1996).

[20] S. Grimme, J. Comput. Chem. 27, 1787 (2006).

[21] N. Atodiresei, V. Caciuc, P. Lazić, and S. Blügel, Phys. Rev. Lett. 102, 136809 (2009).

[22] C. Wittneven, R. Dombrowski, S.H. Pan, and R. Wiesendanger, Rev. Sci. Instrum. 68, 3806 (1997).

[23] A. Kubetzka, M. Bode, O. Pietzsch, and R. Wiesendanger, Phys. Rev. Lett. 88, 057201 (2002).

[24] R. Wiesendanger et al., Phys. Rev. Lett. 65, 247 (1990).

[25] Orientation $A$ is only observed when prepared on a precooled surface, indicating a metastable configuration.

[26] See supplementary material at http://link.aps.org/ supplemental/10.1103/PhysRevLett.105.047204 for additional experimental data.

[27] X. Ge et al., J. Am. Chem. Soc. 131, 6096 (2009).

[28] P. A. Reynolds and B. N. Figgis, Inorg. Chem. 30, 2294 (1991). 